\title{
Effect of alloying BaTiO3 with BiZn1/2Ti1/2O3 on polarization reversal
}

Fancher, C. M.; Choe, H.; Gorfman, S.; Simons, Hugh; Chung, C. C.; Ziolkowski, M.; Prasertpalichat, S.; Cann, D. P.; Jones, J. L.

Published in:

Applied Physics Letters

Link to article, DOI:

$10.1063 / 5.0013410$

Publication date:

2020

Document Version

Publisher's PDF, also known as Version of record

Link back to DTU Orbit

Citation (APA):

Fancher, C. M., Choe, H., Gorfman, S., Simons, H., Chung, C. C., Ziolkowski, M., Prasertpalichat, S., Cann, D. P., \& Jones, J. L. (2020).' Effect of alloying $\mathrm{BaTiO}_{3}$ with $\mathrm{BiZn}_{1} \mathrm{Ti}_{3} \mathrm{O}_{3}$ on polarization reversal. Applied Physics Letters, 117(4), [042907]. https://doi.org/30.1063/5.06234t/ 3

- Users may download and print one copy of any publication from the public portal for the purpose of private study or research.

- You may not further distribute the material or use it for any profit-making activity or commercial gain

- You may freely distribute the URL identifying the publication in the public portal 


\section{Effect of alloying $\mathrm{BaTiO}_{3}$ with $\mathrm{BiZn}_{1 / 2} \mathrm{Ti}_{1 / 2} \mathrm{O}_{3}$ on polarization reversal}

Cite as: Appl. Phys. Lett. 117, 042907 (2020); https://doi.org/10.1063/5.0013410

Submitted: 11 May 2020 . Accepted: 17 July 2020 . Published Online: 30 July 2020

D C. M. Fancher, (D) H. Choe, S. Gorfman, H. Simons, (D) C. C. Chung, M. Ziolkowski, S. Prasertpalichat, (D) D. P. Cann, and (D) J. L. Jones
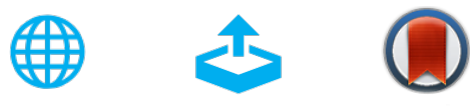

View Online

Export Citation

\section{ARTICLES YOU MAY BE INTERESTED IN}

Stress-modulated optimization of polymorphic phase transition in Li-doped (K, $\mathrm{Na}) \mathrm{NbO}_{3}$ Applied Physics Letters 117, 032901 (2020); https://doi.org/10.1063/5.0016072

Modulation of the electric and magnetic properties by Ti non-stoichiometry in $0.70 \mathrm{BiFeO}_{3}-0.30 \mathrm{BaTi}_{\mathrm{X}} \mathrm{O}_{3}$ ceramics

Applied Physics Letters 117, 042904 (2020); https://doi.org/10.1063/5.0016342

Enhanced intrinsic piezoelectric response in (001)-epitaxial single c-domain $\mathrm{Pb}(\mathrm{Zr}, \mathrm{Ti}) \mathrm{O}_{3}$ nanorods

Applied Physics Letters 117, 042905 (2020); https://doi.org/10.1063/5.0012998

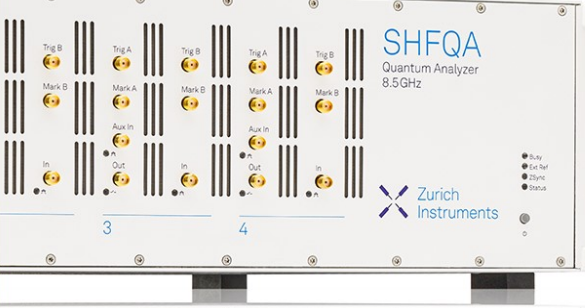

\section{Learn how to perform the readout of up to 64 qubits in parallel With the next generation of quantum analyzers on November 17th




\title{
Effect of alloying $\mathrm{BaTiO}_{3}$ with $\mathrm{BiZn}_{1 / 2} \mathrm{Ti}_{1 / 2} \mathrm{O}_{3}$ on polarization reversal
}

\author{
Cite as: Appl. Phys. Lett. 117, 042907 (2020); doi: 10.1063/5.0013410 \\ Submitted: 11 May 2020 - Accepted: 17 July 2020 . \\ Published Online: 30 July 2020
}

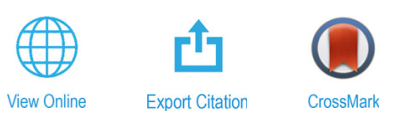

\author{
C. M. Fancher, ${ }^{7}$ (D) H. Choe, ${ }^{2,3}$ (iD S. Gorfman, ${ }^{4}$ H. Simons, ${ }^{5}$ C. C. Chung, ${ }^{6}$ (D) M. Ziolkowski, ${ }^{7}$ S. Prasertpalichat, ${ }^{8}$ \\ D. P. Cann, ${ }^{8}$ (D) and J. L. Jones ${ }^{9, a)}$ (D)
}

\author{
AFFILIATIONS \\ ${ }^{7}$ Neutron Scattering Science Division, Oak Ridge National Laboratory, Oak Ridge, Tennessee 37831, USA \\ ${ }^{2}$ Materials Science and Engineering Division, National Institute of Standards and Technology, Gaithersburg, Maryland 20899, USA \\ ${ }^{3}$ Euclid Techlabs LLC, Bolingbrook, Illinois 60440, USA \\ ${ }^{4}$ Department of Materials Science and Engineering, Tel Aviv University, Tel Aviv 6997801, Israel \\ ${ }^{5}$ Department of Physics, Technical University of Denmark, 2800 Kgs. Lyngby, Denmark \\ ${ }^{6}$ Analytical Instrumentation Facility, North Carolina State University, Raleigh, North Carolina 27606, USA \\ ${ }^{7}$ Department of Physics, University of Siegen, Siegen 57072, Germany \\ ${ }^{8}$ Materials Science, School of Mechanical, Industrial, and Manufacturing Engineering, Oregon State University, Corvallis, \\ Oregon 97331, USA \\ ${ }^{9}$ Department of Materials Science and Engineering, North Carolina State University, Raleigh, North Carolina 27606, USA
}

${ }^{\text {a) }}$ Author to whom correspondence should be addressed: jacob_jones@ncsu.edu

\begin{abstract}
Changes in the polarization state of ferroelectric materials are mediated through the motion of planar defects such as domain walls. The interplay between the two processes that enable the inversion of the macroscopic polarization in ferroelectric materials, non- $180^{\circ}(\mathrm{domain}$ reorientation) and $180^{\circ}$ (domain reversal), is not well understood. In this work, time-resolved x-ray diffraction was carried out during the application of an external electric field to investigate the dynamic electric-field response mechanisms of $\mathrm{BaTiO}_{3}(\mathrm{BT})$ and $\mathrm{BiZn}_{1 / 2} \mathrm{Ti}_{1 / 2} \mathrm{O}_{3}(\mathrm{BZT})-\mathrm{mod}_{-}$ ified BT (0.94BT-0.06BZT). These data evidence that the incorporation of BZT into BT fundamentally alters what processes are activated to reorient the polarization by $180^{\circ}$. Polarization reversal in BT is achieved through direct inversion of spontaneous dipoles. However, the addition of BZT into BT promotes a two-step polarization reversal process (i.e., two consecutive non- $180^{\circ}$ reorientation events).
\end{abstract}

Published under license by AIP Publishing. https://doi.org/10.1063/5.0013410

Polarization and polarization reorientation are central to the functionality of ferroelectric materials. ${ }^{1}$ For example, the macroscopic piezoelectricity in perovskite ferroelectric materials requires a noncentrosymmetric structure and non-random distribution of spontaneous polarization. Changes in the polarization state of ferroelectric materials may be achieved through the motion of planar defects, domain walls (DWs), which separate regions of differing spontaneous polarizations. In ferroelectric materials, two types of domain walls, non- $180^{\circ}$ or $180^{\circ}$, separate domains differing in orientation by either non- $180^{\circ}$ or $180^{\circ}$ rotations, respectively..$^{2-4}$ DWs and domain wall motion (DWM) give rise to non-negligible extrinsic contributions to the macroscopic properties of ferroelectrics, ${ }^{1-8}$ e.g., strong enhancement in their piezoelectric coefficients. While DWM underpins the properties of ferroelectrics, the dynamics of DWM are not well understood. Some theoretical and experimental work has enabled researchers to probe the dynamic electric-field response mechanisms of ferroelectric materials. ${ }^{9-12}$ For example, time-resolved $\mathrm{x}$-ray diffraction (XRD) and piezo-force microscopy (PFM) studies of thin films and bulk ceramics have suggested that the $180^{\circ}$ polarization reversal (domain switching) can be achieved through multiple non- $180^{\circ}$ reorientation events. ${ }^{13-16}$

In this paper, we present a time-resolved in situ x-ray diffraction study that investigates the pathways of polarization reversal in the (1-x) $\mathrm{BaTiO}_{3}-\mathrm{xBiZn}_{1 / 2} \mathrm{Ti}_{1 / 2} \mathrm{O}_{3}$ (BT-BZT) system, a prototype for disordered lead-free ferroelectrics with a transition from classical to relaxor ferroelectric near 0.9BT-0.1BZT. Time-resolved powder diffraction data from BT and 0.94BT-0.06BZT during the application of $50 \mathrm{~Hz}$ cyclic electric fields were measured with a time resolution of 40 $\mu \mathrm{s}$. We demonstrate that the spontaneous polarization in BT is directly inversed during polarization reversal. However, polarization reversal 
in BT modified with 6 mol. \% BZT follows a two-step process (i.e., two consecutive non- $180^{\circ}$ switching events).

A ceramic $0.94 \cdot \mathrm{BaTiO}_{3}-0.06 \cdot \mathrm{BiZn}_{1 / 2} \mathrm{Ti}_{1 / 2} \mathrm{O}_{3}$ sample (herein referred to as $94 \mathrm{BT}$ ) was prepared via conventional solid state synthesis. ${ }^{17}$ After binder burnout at $400^{\circ} \mathrm{C}$ for $3 \mathrm{~h}$, the green pellets were sintered in closed crucibles at $1200^{\circ} \mathrm{C}$ for $2 \mathrm{~h}$ at a heating rate of $3^{\circ} \mathrm{C} /$ min and a cooling rate of $5^{\circ} \mathrm{C} / \mathrm{min}$. Ceramic disks were cut into dimensions suitable for high-energy $\mathrm{x}$-ray diffraction experiment: $0.67 \mathrm{~mm}$ (along the $\mathrm{x}$-ray beam) $\times 8.6 \mathrm{~mm}$ (perpendicular to the $\mathrm{x}$-ray beam) $\times 1.10 \mathrm{~mm}$ (perpendicular to the $\mathrm{x}$-ray beam and parallel to the applied electric field). $\mathrm{BaTiO}_{3}$ powder with an average particle size of $60 \mathrm{~nm}$ (Alfa-Aesar, Ward Hill, MA, USA) was pressed into green compacts, heated in air at $10^{\circ} \mathrm{C} / \mathrm{min}$ to $1350^{\circ} \mathrm{C}$, held for $2 \mathrm{~h}$, and then cooled at a rate of $10^{\circ} \mathrm{C} / \mathrm{min}$ to room temperature. Sintered pellets were cut into $1 \mathrm{~mm}$ (along the applied electric field) $\times 10 \mathrm{~mm}$ (perpendicular to the beam) $\times 0.5 \mathrm{~mm}$ (along the $\mathrm{x}$-ray beam) plates. BT and $94 \mathrm{BT}$ had average grain sizes of $3.00 \pm 0.41 \mu \mathrm{m}$ and $1.45 \pm 0.76 \mu \mathrm{m}$, respectively. ${ }^{18}$ The densities of the BT and 94BT samples were measured using the Archimedes principle, and densities greater than $95 \%$ of theoretical value were achieved after sintering. Both samples were electroded using silver paste for diffraction studies and then electrically poled by repeated electric field cycling.

In situ time-resolved powder diffraction measurements were performed at high-resolution powder diffraction beamline ID22 of the European Synchrotron Radiation Facility (ESRF) at a wavelength of $\lambda=0.413 \AA$ (30.01 keV). ${ }^{19}$ An avalanche photodiode (APD) detector equipped with a $\mathrm{Si}$ (111) analyzer crystal was used to measure diffracted $\mathrm{x}$-rays. A $50 \mathrm{~Hz}$ bipolar positive-up-negative-down (PUND) high-voltage load was applied to the sample using an arbitrary function generator (HMF2550, Hameg, Germany) and a high voltage amplifier (AMT-3B20, Matsusada, Japan). High-resolution diffraction data of 002 as well as the 200 and 020 reflections, which are coincident (herein referred to as the 200 reflection), were measured by stepping the detector arm from $11.25^{\circ}$ to $11.35^{\circ}$ in $2 \theta\left(0.0005^{\circ} /\right.$ step, $40 \mathrm{~s}$ per step). The electric field loading setup was rotated by $\theta=5.7^{\circ}$ to ensure that the measured diffraction data were representative of the powder grains, in which the applied field is parallel to the scattering vector [Fig. 1(a)]. Time-resolved x-ray diffraction data were acquired using a bespoke stroboscopic multi-channel analyzer data acquisition system (10 000 data channels). ${ }^{20-26}$ Measured data were later binned by a factor of 20 to improve the counting statistics, resulting in a final time resolution of $40 \mu \mathrm{s}$. The electric current was monitored using an active probe measuring the voltage drop across a $1 \mathrm{k} \Omega$ active resistor with a resolution of $6 \mu$ s using a Rohde and Schwarz oscilloscope (HMO3044, Hameg, Germany).

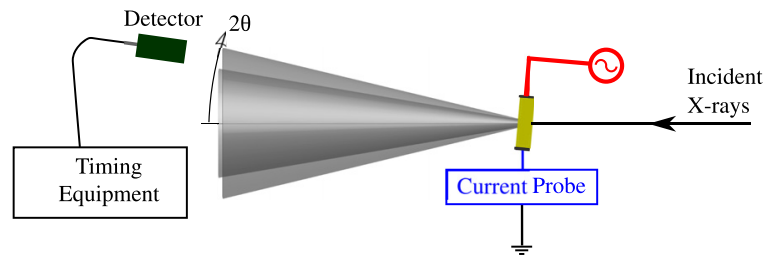

FIG. 1. Schematic representation of the experimental setup used to measure timeresolved diffraction data. The detector signal was parsed using a multi-channel analyzer that facilitated the measurement of time-dependent data.
Time-resolved diffraction enables the direct interrogation of the structural evolution of materials in response to an externally applied stimulus as a function of time. Systematic changes in the measured diffraction data provide vital insight into what electric field response mechanisms are activated and in the order in which these mechanisms are activated. Figure 2 shows the measured time- and field-dependent 002 and 200 reflections of BT (a) and 94BT (b). Variations in the intensity of the 002 and 200 reflections enable a direct quantification of non- $180^{\circ} \mathrm{DWM}$ in materials with a tetragonal symmetry (e.g., BT and 94BT). BT exhibits an increase in the intensity of the 002 reflection during the application of the PUND waveform. In contrast, 94BT first exhibits a drastic reduction in the intensity of 002 with a commensurate increase in 002 during the switching pulse, while the second positive or negative field excursion of similar polarity only induces ferroelastic switching, reorienting dipoles by $90^{\circ}$ into the field direction. The observation of an initial reduction in the intensity of 002 shows that the spontaneous polarization does not directly follow the electric field; instead, a significant volume fraction of domains reorients their polarization perpendicular to the applied field before reorienting again to follow the electric field. The stark difference in the evolution in the diffraction signal of BT and 94BT during the first positive and negative pulses suggests that each material activates different mechanism(s) when strong electric fields are applied antiparallel to the macroscopic polarization state. It should be noted that no evidence for the coexistence of the $P 4 m m$ and $P m \overline{3} m$ mixture is observed in the present samples with or without the application of electric fields. ${ }^{9,27}$ Only subtle variations in the total integrated intensity per time channel, which are ascribed to anomalous scattering, are measured (see Figs. S1 and S2).

The measured diffraction data were further analyzed via single peak fitting to extract the position and intensity of the 002 and 200 reflections. ${ }^{28,29}$ Extracted peak intensities were used to determine the change in the fraction of dipoles oriented in the field direction using the following relation: ${ }^{30}$

$$
\eta_{002}=\frac{\frac{I_{002}}{I_{002}^{\prime}}}{\frac{I_{002}}{I_{002}^{\prime}}+2 \frac{I_{200 / 020}}{I_{200 / 020}^{\prime}}}-\frac{1}{3},
$$

where $I_{00 h}$ is the integrated intensity of $00 h$ reflection in an electrically poled state and $I_{00 h}^{\prime}$ is the integrated intensity of the $00 \mathrm{~h}$ reflection with a random distribution of domains (i.e., has not experienced electric fields). The ratio of $\frac{I_{200 / 202}^{\prime}}{I_{002}^{\prime}}$ is $\sim 1.99$ for both BT and 94BT. The intensity for a randomly oriented material was obtained from virgin samples of each composition. Representative fits for both BT and 94BT are shown in the supplementary material, Fig. S1. The evolution in the volume fraction of domains aligned in the field direction during the PUND waveform is different between BT and 94BT, highlighting their unique domain switching mechanisms. While both BT and 94BT exhibit an enhancement in $\eta_{002}$ during the field pulses, $\eta_{002}$ dips below 0 during the drastic intensity interchange observed in 94BT for the first pulses of the opposite polarity. A negative $\eta_{002}$ indicates a state in which a larger volume fraction of domains with the $\mathrm{c}$-axis perpendicular to the field than that is present in an unpoled sample. The observation of a negative $\eta_{002}$ during field reversal is direct evidence that the spontaneous dipoles in 94BT first reorient by $90^{\circ}$ to preferentially 

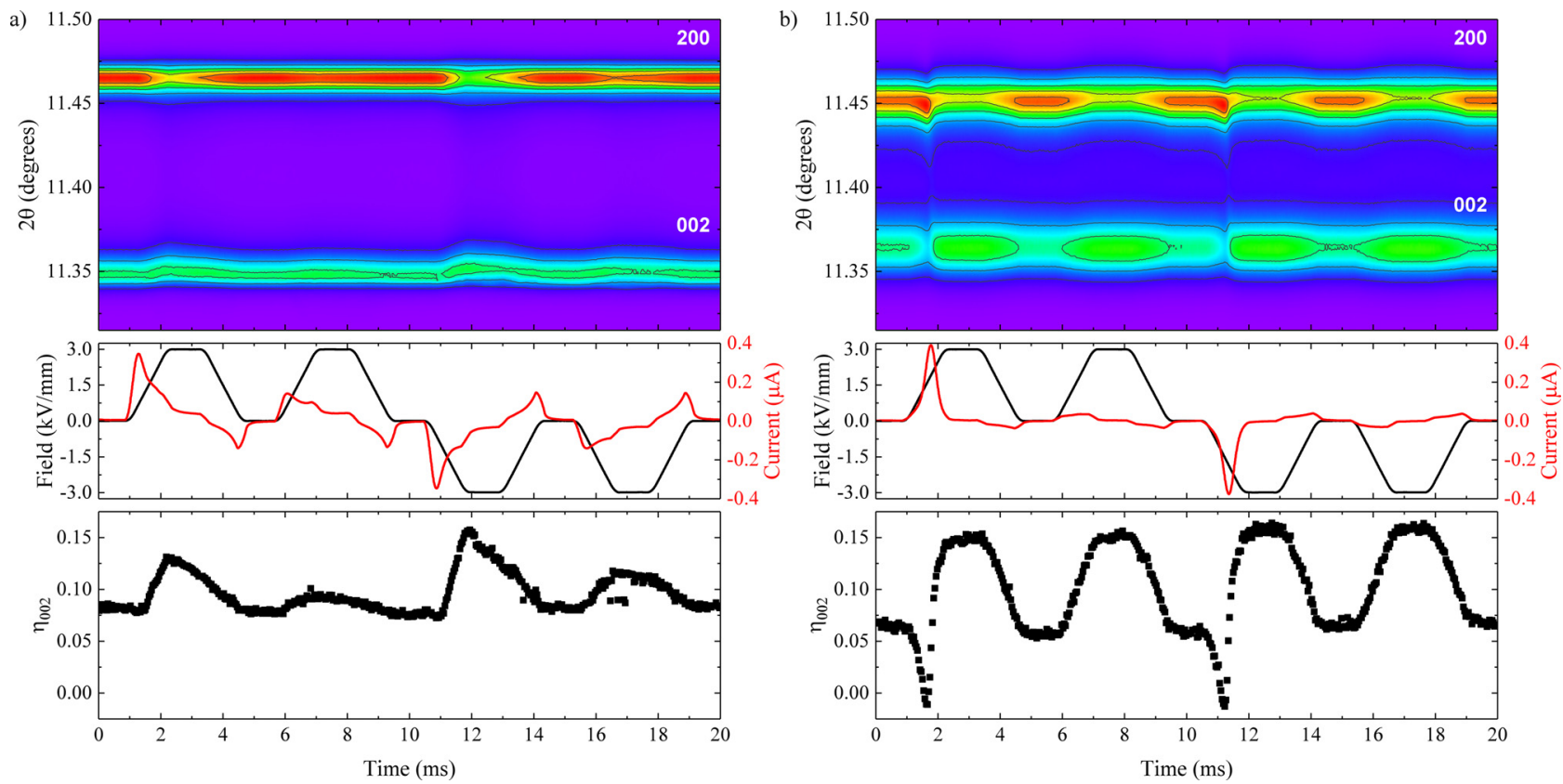

FIG. 2. Time-dependent diffraction data, the fraction of domains aligned in the field direction $\left(\eta_{002}\right)$ and the current signal to an applied PUND waveform for BT (a) and 94BT (b).

populate states perpendicular to the applied electric field before reorienting again to align with the electric field.

We note that BT and 94BT also exhibit different changes in $\eta_{002}$ during the $\mathrm{U}$ and $\mathrm{D}$ segments of the PUND waveform. Each field pulse induces a nearly equivalent $\eta_{002}$ in 94BT, which remains constant throughout the pulse. BT acts differently, with $\eta_{002}$ being maximized at the onset of the $\mathrm{P}$ and $\mathrm{N}$ pulses followed by a decay during the constant field hold. Subsequent applications of the $U$ and $D$ pulses do not induce the same extent of domain alignment in the field direction as the $\mathrm{P}$ and $\mathrm{N}$ pulses. The decay in $\eta_{002}$ is accompanied by a decaying non-zero current signal (in the field direction), which indicates that further $180^{\circ} \mathrm{DWM}$ is induced while holding at a constant field. We attribute this $180^{\circ} \mathrm{DWM}$ to sluggish kinetics and/or domains that require a higher applied electric field to overcoming pinning. ${ }^{31,32}$ The decay in $\eta_{002}$ indicates that $90^{\circ}$ back switching occurs to accommodate changes in the local electric/mechanical energy with the additional $180^{\circ} \mathrm{DWM}$ with the constant field hold. One explanation is that the induced $180^{\circ} \mathrm{DWM}$ during the constant field hold increases the intergranular stress due to the difference in the piezoelectric effect between neighboring grains. ${ }^{33-36}$ The sluggish nature of the late stage $180^{\circ}$ DWM induced during the $\mathrm{P}$ and $\mathrm{N}$ pules might lock in a residual stress that inhibits $90^{\circ}$ DWM during the U and D pulses. ${ }^{15}$

Unlike measured diffraction intensities that are predominantly insensitive to breaking of inversion symmetry, the electrical signal of the BT and 94BT samples is sensitive to both domain reorientation and reversal. Correlating the current signal measured simultaneously with diffraction data enables the identification of the specific switching mechanism $\left(180^{\circ}\right.$ or non- $\left.180^{\circ}\right)$ that gives rise to the respective current signal. Similar to the diffraction data, the electric current through BT and 94BT differs drastically, further indicating that different domain switching mechanisms are activated during the application of PUND electric fields. A single inflection in current is observed on the first positive and negative field pulses for 94BT, suggesting that the macroscopic polarization of $94 \mathrm{BT}$ is reversed over a narrow range of electric fields. A close comparison of the current signal and the change in domains aligned in the field direction for the $\mathrm{P}$ and $\mathrm{N}$ segments of the PUND waveform is shown in fig. S4. The minima in domains aligned in the field direction are found to occur 0.110 and $0.138 \mathrm{~ms}$ prior to the maximum/minimum in the current signal for the $\mathrm{P}$ and $\mathrm{N}$ segments, respectively. The change in polarization data was modeled using the methods reported by Genenko et al. to extract the switching times for the $90^{\circ}$ domain reorientation and $180^{\circ}$ domain reversal (see the supplementary material). ${ }^{37}$ These results indicate that the switching of the perpendicular domains into the field direction $(0.87 \mathrm{~ms})$ has a similar switching time as the $180^{\circ}$ domain reversal $(0.91 \mathrm{~ms})$. BT exhibits a more complex current signal than 94BT, with two inflections that indicate activation of multiple mechanisms. No change in the diffraction intensities is observed for the first current inflections, while the second occurs simultaneously with an increase in $\eta_{002}$, evidencing that $180^{\circ}$ reversal occurs at a lower electric field than of the non- $180^{\circ}$ case. This observation agrees with recent work that modeled the evolution of the total dipole distribution function of BT with applied fields. ${ }^{38}$ Evidence for both a single 180 and multiple $90^{\circ}$ switching has been observed in single crystals of BT. ${ }^{39,40} \mathrm{Li}$ and Li hypothesized that interactions between neighboring domains could provide sufficient mechanical clamping to inhibit the nucleation of $90^{\circ}$ domains. ${ }^{39}$ In the context of polycrystalline ceramics, intergranular stresses provide an additional source that could mechanically clamp $90^{\circ}$ domains.

Correlating the diffraction and electric current data enables the determination of mechanisms that are activated to achieve polarization reversal in BT and 94BT, which is schematically represented in Fig. 3. The domain reversal mechanisms are summarized in Fig. 3, which 
BT
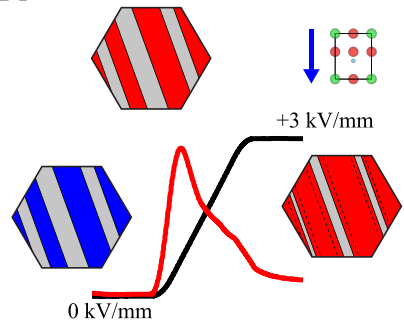

94BT
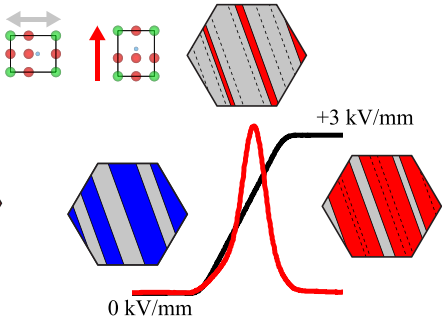

FIG. 3. Schematic of the pathway of polarization reversal for BT and BT-BZT. Domains are colored to indicate their polarization state. Blue, gray, and red regions represent domains that have a negative polarization, polarization perpendicular to the applied field, and positive polarization states, respectively. Negative and positive polarization states represent spontaneous polarizations that are oriented antiparallel and parallel to the scattering vector, respectively. Both BT and 94 BT start and end with a common domain structure. The intermediate reversal processes of BT and 94 BT differ. Dashed lines are shown to indicate the initial position of the DW.

schematically depicts the evolution of the domains for the $\mathrm{P}$ and $\mathrm{N}$ pulses of the PUND waveform where the macroscopic polarization is being inverted. Negative and positive polarization states represent spontaneous polarizations that are oriented antiparallel and parallel to the scattering vector, respectively. The microstructure is initially dominated by domains that are oriented in the "Down" or negative state, as defined by the direction with respect to the scattering vector. In BT (Fig. 3), the negative domains are first reversed into positive domains, and then a volume fraction of domain oriented perpendicular to the field is reoriented into positive domains. The drastic reduction in the intensity of 002 in 94 BT (Fig. 3) evidences that the negative domains are reoriented by $90^{\circ}$ into a metastable configuration perpendicular to the field before undergoing a second reorientation into positive domains.

Diffraction signatures of a non- $180^{\circ}$ domain reorientation mechanism were initially reported for $\mathrm{PbZr}_{\mathrm{x}} \mathrm{Ti}_{1-\mathrm{x}} \mathrm{O}_{3}$ in 1993 by $\mathrm{Li}$ et al. ${ }^{41}$ Evidence for a two-step reversal process was not observed until 2007 in $0.955 \mathrm{~Pb}\left(\mathrm{Zn}_{1 / 3} \mathrm{Nb}_{2 / 3}\right) \mathrm{O}_{3}-0.045 \mathrm{PbTiO}_{3}$ (PZN-PT). ${ }^{13}$ More recent experimental $^{14}$ and theoretical ${ }^{37}$ studies have suggested that non- $180^{\circ}$ DWM is the first switching mechanism activated in ferroelectric materials. Schultheiß proposed that the application of electric fields antiparallel to the polarization directions activates a sequence of switching processes: 1) non- $180^{\circ} \mathrm{DWM}, 2$ ) cascade of both non- $180^{\circ}$ and $180^{\circ}$ switching events, and 3) further non- $180^{\circ}$ DWM (creep response). These data indicated that 94BT does undergo a similar set of processes; however, BT only shows the signature of non- $180^{\circ}$ DWM after $180^{\circ}$ reversal, as indicated by the electrical current maxima.

Observing a different sequence of switching mechanisms in BT and 94BT should originate from differences in the microstructure or crystallography of the two material systems. The grain sizes of BT $(3.00 \pm 0.41 \mu \mathrm{m})$ and 94BT $(1.45 \pm 0.76 \mu \mathrm{m})$ could result in different initial domain wall densities. Specifically, in BT, the difference in the grain size would impact the piezoelectric properties, $\mathrm{P}_{\mathrm{r}}$, and $\mathrm{E}_{\mathrm{c}}{ }^{42-44}$ For example, reducing the grain size from 3 to $1.5 \mu \mathrm{m}$ has been shown to increase $\mathrm{E}_{\mathrm{c}}$ from 0.15 to $0.175 \mathrm{kV} / \mathrm{mm} .{ }^{42}$ However, alloying BT with $0.06 \mathrm{BZT}$ has a far more pronounced impact on the properties of BTBZT than what is observed from grain size effects. ${ }^{17}$ Thus, the differences in the sequence of switching mechanisms might originate from the underlying crystallography of each material system. For example, Landau-Ginzburg-Devonshire theory suggests that the origin of the superior properties near the morphotropic phase boundary (MPB) arises from a flattening of the energy landscape where multiple phases have comparable free energies. ${ }^{45}$ While $\mathrm{PbZr}_{0.2} \mathrm{Ti}_{0.8} \mathrm{O}_{3}$ is far from the $\mathrm{MPB}$, epitaxial strain can influence the switching behavior of ferroelectric materials. ${ }^{46}$ Instead of an MPB, 94BT is in close proximity to the ferroelectric-relaxor transition near $8 \mathrm{~mol}$. \% BZT, which might arise due to the destabilization of the long-range order by oxygen vacancies induced by alloying BT with BZT. ${ }^{47}$ Pair distribution functions (PDFs) and Rietveld analysis have demonstrated that 94BT has differing localand long-range ordering, where Bragg diffraction has only suggested a pseudocubic structure that obscures the local structure. ${ }^{18,27}$ The authors used Small-box modeling to demonstrate that the BT-BZT has short range tetragonal distortion that decays toward a psuedocubic with the increasing length scale, finding that the onset length scale decreases with the increasing BZT content. Evidence for a phase coexistence of tetragonal and psuedocubic phases is observed near the relaxor transitions. ${ }^{24}$ While BT has been shown to localize Ti-O bonding at the 1-2 unit cell length with $\mathrm{Ti}^{4+}$ distortions along $\langle 111\rangle$, these distortions average to a $\langle 001\rangle$ distortion in the medium and long range and are best described using a single phase $P 4 \mathrm{~mm}$ structure model. ${ }^{48,49}$ The observation of a two-step domain reorientation process in BZTBT (94BT) near a relaxor transition, as well as in relaxor ferroelectric PZN-PT and PZT near the MPB, suggests that the local structure and/ or phase coexistence might play a significant role in governing the dynamics of DWM and polarization reversal.

In conclusion, time-resolved diffraction was used to study the pathway for polarization reversal in pure BT and 94BT-6BZT. These results evidence that the change in the chemical composition and local structure can greatly influence how the inversion of a spontaneous polarization is achieved. Combined analysis of Bragg intensities and electric current data suggests that polarization in BT reverts through spontaneous dipoles. In contrast, 94BT exhibits a more complex domain-switching process that first undergoes an intermediate non$180^{\circ}$ switching that is followed by both $180^{\circ}$ domain reversal and non- $180^{\circ}$ domain reorientation.

See the supplementary material for sample representative fits and total intensity as a function of applied field.

The users acknowledge the ESRF for providing the beamtime through the user proposal system. C.M.F. and J.L.J. acknowledge support for this work from the National Science Foundation, as part of the Center for Dielectrics and Piezoelectrics under Grant Nos. IIP-1361571 and IIP-1361503. C.M.F acknowledges support at the Oak Ridge National Laboratory by the DOE Office of Science User Facilities. H.S. acknowledges a European Research Council starting Grant No. \#804665. S.G. acknowledges support of the Israel Science Foundation (Grant No 51/5651). This manuscript was authored by UT-Battelle, LLC under Contract No. DE-AC0500OR22725 with the U.S. Department of Energy. The United States Government retains and the publisher, by accepting this article for publication, acknowledges that the United States Government retains a non-exclusive, paid-up, irrevocable, and worldwide license to publish or reproduce the published form of this manuscript, or allow others to do so, for United States Government purposes. The 
Department of Energy will provide public access to these results of federally sponsored research in accordance with the DOE Public Access Plan (http://energy.gov/downloads/doe-public-access-plan).

\section{DATA AVAILABILITY}

The data that support the findings of this study are available from the corresponding author upon reasonable request.

\section{REFERENCES}

${ }^{1}$ L. E. Cross, Mater. Chem. Phys. 43, 108 (1996).

${ }^{2}$ M. C. Ehmke, N. H. Khansur, J. E. Daniels, J. E. Blendell, and K. J. Bowman, Acta Mater. 66, 340 (2014).

${ }^{3}$ J. E. Daniels, W. Jo, J. Rödel, V. Honkimäki, and J. L. Jones, Acta Mater. 58, 2103 (2010).

${ }^{4}$ J. L. Jones, M. Hoffman, J. E. Daniels, and A. J. Studer, Appl. Phys. Lett. 89, 092901 (2006).

${ }^{5}$ D. Ghosh, A. Sakata, J. Carter, P. A. Thomas, H. Han, J. C. Nino, and J. L. Jones, Adv. Funct. Mater. 24, 885 (2014).

${ }^{6}$ A. Pramanick, D. Damjanovic, J. E. Daniels, J. C. Nino, and J. L. Jones, J. Am. Ceram. Soc. 94, 293 (2011).

${ }^{7}$ D. Damjanovic, J. Appl. Phys. 82, 1788 (1997).

${ }^{8}$ D. A. Hall, J. Mater. Sci. 36, 4575 (2001).

${ }^{9}$ T. Iamsasri, G. Esteves, H. Choe, M. Vogt, S. Prasertpalichat, D. P. Cann, S. Gorfman, and J. L. Jones, J. Appl, Phys. 122, 64104 (2017).

${ }^{10}$ Y. A. Genenko, S. Zhukov, S. V. Yampolskii, J. Schütrumpf, R. Dittmer, W. Jo, H. Kungl, M. J. Hoffmann, and H. Von Seggern, Adv. Funct. Mater. 22, 2058 (2012).

${ }^{11}$ S. Zhukov, Y. A. Genenko, O. Hirsch, J. Glaum, T. Granzow, and H. von Seggern, Phys. Rev. B 82, 014109 (2010).

${ }^{12}$ R. Khachaturyan, J. Wehner, and Y. A. Genenko, Phys. Rev. B 96, 054113 (2017).

${ }^{13}$ J. E. Daniels, T. R. Finlayson, M. Davis, D. Damjanovic, A. J. Studer, M. Hoffman, and J. L. Jones, J. Appl. Phys. 101, 104108 (2007).

${ }^{14}$ J. Schultheiß, L. Liu, H. Kungl, M. Weber, L. Kodumudi Venkataraman, S. Checchia, D. Damjanovic, J. E. Daniels, and J. Koruza, Acta Mater. 157, 355 (2018).

${ }^{15}$ J. E. Daniels, C. Cozzan, S. Ukritnukun, G. Tutuncu, J. Andrieux, J. Glaum, C. Dosch, W. Jo, and J. L. Jones, J. Appl. Phys. 115, 224104 (2014).

${ }^{16}$ R. Xu, S. Liu, I. Grinberg, J. Karthik, A. R. Damodaran, A. M. Rappe, and L. W. Martin, Nat. Mater. 14, 79 (2015).

${ }^{17}$ C.-C. Huang and D. P. Cann, J. Appl. Phys. 104, 024117 (2008).

${ }^{18}$ D. Hou, T. M. Usher, H. Zhou, N. Raengthon, N. Triamnak, D. P. Cann, J. S. Forrester, and J. L. Jones, J. Appl. Phys. 122, 064103 (2017).

${ }^{19}$ A. N. Fitch, J. Res. Natl. Inst. Stand. Technol. 109, 133 (2004).

${ }^{20}$ S. Gorfman, O. Schmidt, V. G. Tsirelson, M. Ziolkowski, and U. Pietsch, Z. Anorg. Allg. Chem. 639, 1953 (2013).

${ }^{21}$ S. Gorfman, O. Schmidt, M. Ziolkowski, M. Von Kozierowski, and U. Pietsch, J. Appl. Phys. 108, 064911 (2010).

${ }^{22}$ S. Gorfman, Crystallogr. Rev. 20, 210 (2014).
${ }^{23}$ H. Choe, S. Gorfman, S. Heidbrink, U. Pietsch, M. Vogt, J. Winter, and M. Ziolkowski, IEEE Trans. Nucl. Sci. 64, 1320 (2017).

${ }^{24}$ S. Gorfman, H. Choe, V. V. Shvartsman, M. Ziolkowski, M. Vogt, J. Strempfer, T. Łukasiewicz, U. Pietsch, and J. Dec, Phys. Rev. Lett. 114, 097601 (2015).

${ }^{25}$ B. Khanbabaee, E. Mehner, C. Richter, J. Hanzig, M. Zschornak, U. Pietsch, H. Stöcker, T. Leisegang, D. C. Meyer, and S. Gorfman, Appl. Phys. Lett. 109, 222901 (2016).

${ }^{26}$ H. Choe, J. Bieker, N. Zhang, A. M. Glazer, P. A. Thomas, and S. Gorfman, IUCrJ 5, 417 (2018).

${ }^{27}$ T.-M. Usher, T. Iamsasri, J. S. Forrester, N. Raengthon, N. Triamnak, D. P. Cann, and J. L. Jones, J. Appl. Phys. 120, 184102 (2016).

${ }^{28}$ G. Esteves, K. Ramos, C. M. Fancher, and J. L. Jones, "LIPRAS: Line-Profile Analysis Software," Technical Report (2017).

${ }^{29}$ G. Esteves, C. M. Fancher, and J. L. Jones, J. Mater. Res. 30, 340 (2015).

${ }^{30}$ J. L. Jones, E. B. Slamovich, and K. J. Bowman, J. Appl. Phys. 97, 034113 (2005).

${ }^{31}$ G. Tutuncu, D. Damjanovic, J. Chen, and J. L. Jones, Phys. Rev. Lett. 108, 177601 (2012).

${ }^{32}$ L. Goncalves-Ferreira, S. A. T. Redfern, E. Artacho, E. Salje, and W. T. Lee, Phys. Rev. B 81, 1 (2010).

${ }^{33}$ H. Simons, A. B. Haugen, A. C. Jakobsen, S. Schmidt, F. Stöhr, M. Majkut, C. Detlefs, J. E. Daniels, D. Damjanovic, and H. F. Poulsen, Nat. Mater. 17, 814 (2018).

${ }^{34}$ A. Haug, P. R. Onck, and E. Van der Giessen, Int. J. Solids Struct. 44, 2066 (2007).

${ }^{35}$ S. Choudhury, Y. L. Li, C. Krill III, and L. Q. Chen, Acta Mater. 55, 1415 (2007).

${ }^{36}$ M. Majkut, J. E. Daniels, J. P. Wright, S. Schmidt, J. Oddershede, and J. Ihlefeld, J. Am. Ceram. Soc. 100, 393 (2017).

${ }^{37}$ Y. A. Genenko, R. Khachaturyan, J. Schultheiß, A. Ossipov, J. E. Daniels, and J. Koruza, Phys. Rev. B 97, 144101 (2018).

${ }^{38}$ C. M. Fancher, S. Brewer, C. C. Chung, S. Röhrig, T. Rojac, G. Esteves, M. Deluca, N. Bassiri-Gharb, and J. L. Jones, Acta Mater. 126, 36 (2017).

${ }^{39}$ Y. W. Li and F. X. Li, Appl. Phys. Lett. 104, 229901 (2014).

${ }^{40}$ B. Jiang, Y. Bai, W. Chu, Y. Su, and L. Qiao, Appl. Phys. Lett. 93, 152905 (2008).

${ }^{41}$ S. Li, C.-Y. Huang, A. S. Bhalla, and L. E. Cross, Ferroelectr. Lett. Sect. 16, 7 (1993).

${ }^{42}$ Y. Huan, X. Wang, J. Fang, and L. Li, J. Eur. Ceram. Soc. 34, 1445 (2014).

${ }^{43}$ Z. Zhao, V. Buscaglia, M. Viviani, M. T. Buscaglia, L. Mitoseriu, A. Testino, M. Nygren, M. Johnsson, and P. Nanni, Phys. Rev. B 70, 1 (2004).

${ }^{44}$ G. Arlt, D. Hennings, and G. De With, J. Appl. Phys. 58, 1619 (1985).

${ }^{45}$ D. Damjanovic, Appl. Phys. Lett. 97, 062906 (2010).

${ }^{46}$ J. H. Haeni, P. Irvin, W. Chang, R. Uecker, P. Reiche, Y. L. Li, S. Choudhury, W. Tian, M. E. Hawley, B. Craigo, A. K. Tagantsev, X. Q. Pan, S. K. Streiffer, L. Q. Chen, S. W. Kirchoefer, J. Levy, and D. G. Schlom, Nature 430, 758 (2004).

${ }^{47}$ N. Kumar, E. A. Patterson, T. Frömling, E. P. Gorzkowski, P. Eschbach, I. Love, M. P. Müller, R. A. De Souza, J. Tucker, S. R. Reese, and D. P. Cann, J. Am. Ceram. Soc. 101, 2376 (2018).

${ }^{48}$ J. R. Neilson and T. M. McQueen, J. Appl. Crystallogr. 48, 1560 (2015).

${ }^{49}$ M. S. Senn, D. A. Keen, T. C. A. Lucas, J. A. Hriljac, and A. L. Goodwin, Phys. Rev. Lett. 116, 207602 (2016). 